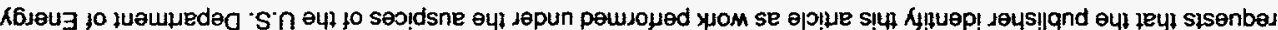

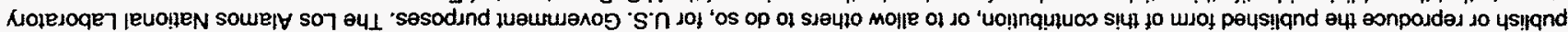

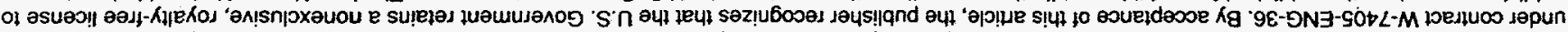

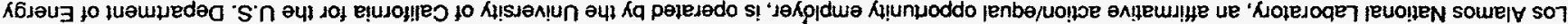

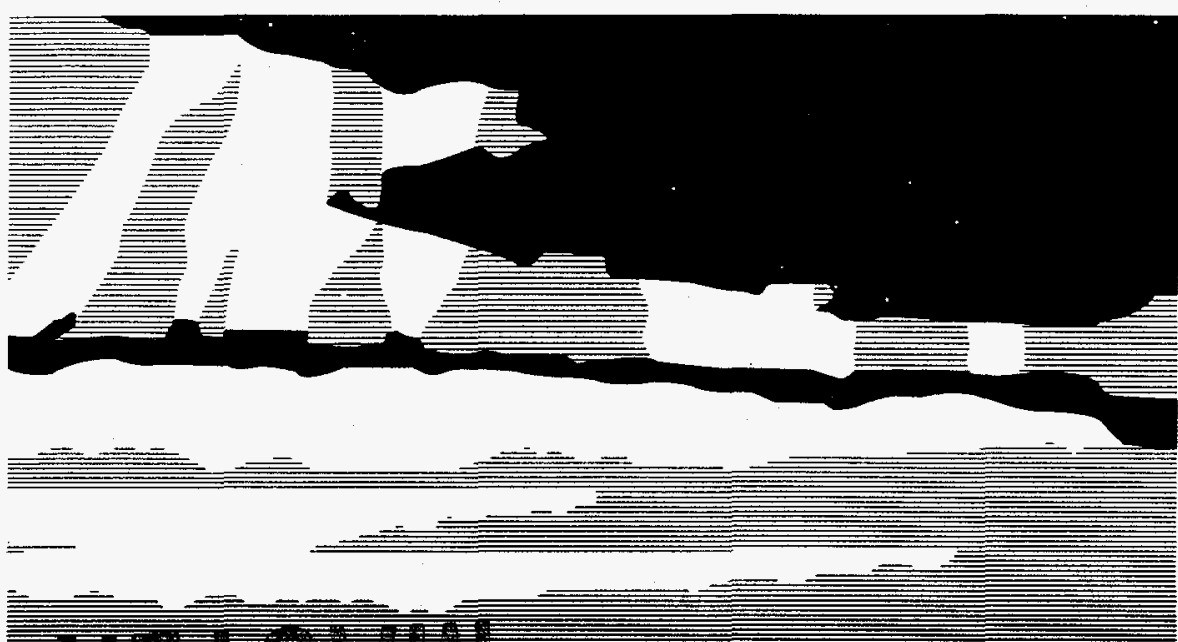

प्ISWW

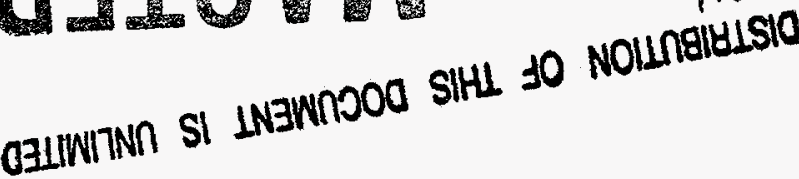

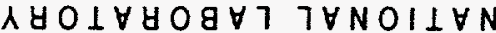 Somel 8 SO7}

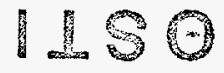

$503620 \mathrm{sin}$

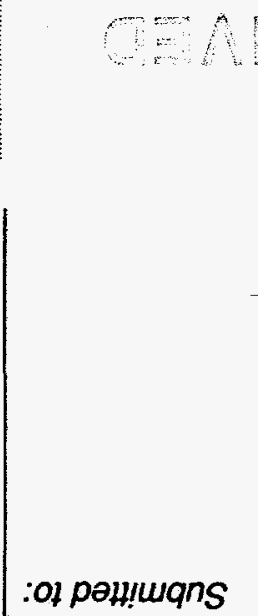

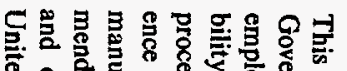

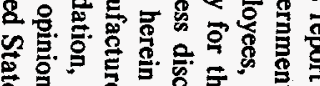

总

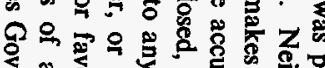

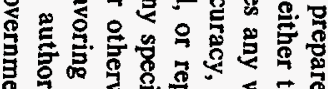

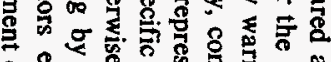

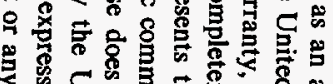

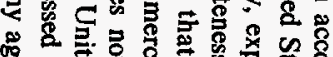

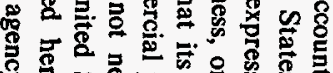

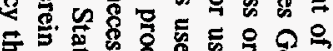

웅

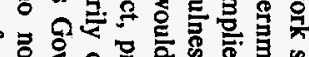

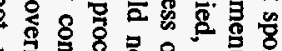

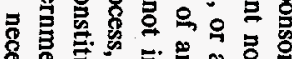

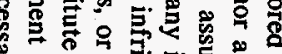

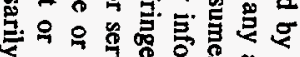

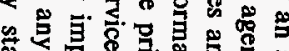

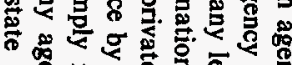

융

唡

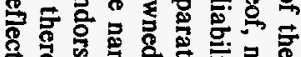

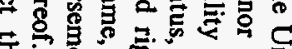

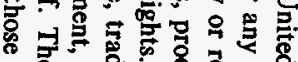

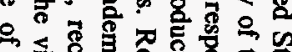

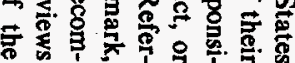

XIITTIRHLS GSHHA

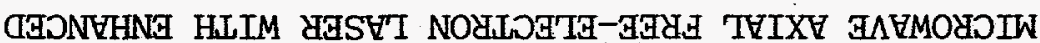

:2|H! 


\section{DISCLAIMER}

Portions of this document may be illegible in electronic image products. Images are produced from the best available original document. 


\title{
MICROWAVE AXIAL FREE-ELECTRON LASER WITH ENHANCED PHASE STABILTY
}

\author{
Bruce E. Caristen, Clifford M. Fortgang, James M. Potter,* \\ Michael V. Fazio, W. Brian Haynes, and Lisa M. May
}

\author{
Los Alamos National Laboratory, Los Alamos, NM 87545 USA \\ *JP Accelerator Works, Inc., Los Alamos, NM 87544 USA
}

Free-electron lasers (FELs) amplifiers have demonstrated high efficiencies and high output power at microwave wavelengths. However, measurements and simulations have indicated that the present level of phase stability for these devices is not sufficient for driving linear accelerators. Fluctuations in the diode voltage, which is needed to accelerate the electron beam, are the largest cause of the shifts in the phase of the output power. Present-day pulse-power technology cannot keep the voltage fluctuations less than $1 / 4 \%$. However, we have found a scheme that will make the output phase much less sensitive to these fluctuations by exploiting the traveling-wave nature of the FEL interaction. In this paper we study the phase stability issue by analyzing the dispersion relation for an axial FEL, in which the rf field is transversely wiggled and the electron trajectories are purely longitudinal. The advantage of using the axial FEL interaction instead of the common transverse FEL interaction is that the dispersion relation is not additionally complicated by how the transverse electron motion depends on the diode voltage and such a device is simpler and less expensive to construct than a transverse-coupling FEL because there is no wiggler. By examination of the dispersion relation it is found that the effect of the phase dependency on the beam's velocity can be cancelled by the effect of the phase dependency on the beam's plasma wave, for an annular electron beam. This cancellation leads to first-order phase stability, which is not possible for standing-wave devices, such as klystrons. Detailed particle-in-cell simulations are included to demonstrate the transverse wiggling of the rf mode and the axial FEL interaction. 


\section{Introduction}

Free-electron lasers (FELs) have demonstrated both high beam-to-rf power extraction efficiencies $(-30 \%)$ [1] and high output power (on the order of gigawatts) [2]. They have, however, also demonstrated poor phase stability (on the order of $20^{\circ}$ to $40^{\circ}$ shift per percent voltage fluctuation $[3,4,5])$. In this paper, we outline a scheme that will drastically improve the FEL phase stability, to a point exceeding that of klystrons.

We propose to use the axial FEL interaction [6], in which the electron beam interacts with a $\mathrm{TM}_{0 m}$ mode in a circular waveguide. The mode is forced to expand and contract in the radial direction by periodically rippling the waveguide radius. As a result, sychronism is established by "wiggling" the rf instead of the electron beam, and no separate wiggler is needed to modify the electrons' orbits. This interaction was chosen for three reasons: (1) the absence of a wiggler simplifies cost and construction of the device, (2) the transverse electric and magnetic forces on the electron beam tend to cancel each other better if the rf is a TM mode as opposed to a TE mode [6], and (3) there are no hidden correlations between the interaction strength and the beam voltage (as there would be if the beam interacts through transverse motions induced via a wiggler), simplifying the phase stability scheme.

The phase-stability scheme is based on setting up a correlation between the space-charge wave on the electron beam and the transit time effect of the beam. Ignoring space charge, the phase evolution of the growing mode in the exponential growth regime is a function of the beam velocity, which changes if the beam voltage fluctuates. However, the phase evolution of an FEL in the Raman regime also depends on the space-charge wave propagating in the beam. By proper design, the phase fluctuations from these two effects can be made to cancel.

In the next section of this paper, we will describe the axial FEL interaction in detail. We will present particle-in-cell (PIC) simulations of the if mode propagation to demonstrate the effective wiggling of the rf mode by its periodic expansion and contraction, for both a long and a short ripple period. Also in this section we will show PIC simulations demonstrating bunching 
and energy extraction from the electron beam, as well as one-dimensional simulations of the particles' longitudinal phase space. In the following section, we will discuss the phase stability mechanism, starting with the dispersion relation which can be solved for the mode's axial evolution. The output phase of the if depends on the imaginary component of the mode's exponential growth constant, which depends both the beam's velocity directly and on the detuning from resonance and the space-charge wave in the beam, which are also functions of the beam energy. By proper manipulation of the beam parameters, we will find that the imaginary component of the mode's growth constant can be made independent of beam energy, leading to enhanced phase stability.

\section{Axial FEL Interaction}

In this section, we outline the main features of the axial FEL interaction and present PIC simulations demonstrating them. In particular, we verify the periodic mode contraction and expansion as it drifts axially, trapping in the pondermotive bucket, energy extraction, and exponential growth of the mode.

In the axial FEL, the electron beam is annular, and the motion is purely axial. The beam interacts with the axial electric field of the $\mathrm{TM}_{0 m}$ mode in a circular waveguide. The radius of this waveguide is periodically rippled which causes the mode to radially expand and contract. The ripple amplitude is only a few percent of the average radius, and the mode is able to adiabatically conform to the gradual change in the waveguide radius. The axial FEL interaction for a synchronous particle is shown in Fig. 1. In the figure, the annulus is located at a radius corresponding to a zero of the axial electric field of that mode in an unrippled waveguide with radius equal to the mean radius of the rippled waveguide. When an electron is at the axial position of the smallest waveguide radius the axial electric field at the location of the electron opposes the electron's motion. As the electron travels to the region of larger radius, the rf slips by the electron (because the mode's phase velocity always exceeds the speed of light). When the electron is at the location of the maximum radius one half rf wavelength has slipped by, resulting in a sign change in the mode's fields. Additionally, the electron is experiencing the electric field at a radius smaller 
than the axial null instead of at a larger radius. This switch from one side of the null of the axial field to the other provides another sign change in the axial field at the location of the electron, and the electric field is again opposing the electron's motion. This interaction is equivalent to the interaction of a transverse-coupling FEL except the rf field is wiggled instead of the electrons to provide synchronism.

If the ripple period is sufficiently long, the rf mode essentially breathes in and out, and the axial if field is given by

$$
E_{z}=A J_{o}\left(k_{c} r /\left(1+\frac{\Delta}{a} \cos \left(k_{w} z\right)\right)\right) e^{j(\omega t-k z)}
$$

where $A$ is some constant, $k_{c}$ is the mode cutoff wavenumber, $\omega$ is the mode frequency, $a$ is the beam pipe radius, $k_{w}=2 \pi / \lambda_{w}$ is the ripple wavenumber ( $\lambda_{w}$ is the ripple period), $J_{o}$ is the zero order Bessel function, $k$ is mode's propagation constant, given by $k^{2}=\omega^{2} / c^{2}-k_{c}^{2}$, and $\Delta$ is the ripple amplitude [6]. If the ripple period is too short, the ripples will effectively act like a slow wave structure, and various space harmonics will be introduced, with the characteristic $I_{o}$ Bessel function radial dependence of the slow-wave harmonics. We will see these limits shortly in our examples.

Although slow-wave space harmonics can interact favorably with an electron beam (this is the basis for all conventional traveling-wave tubes), there are some distinct advantages of the axial FEL interaction where the interaction is with the fast wave. First, essentially all of the power in the mode is transmitted through the structure, and there is no power trapped between the wiggles (which could lead to breakdown for high-power operation). Also, there are no additional spaceharmonics to deal with, that could compete with the desired mode at saturation. In addition, the interaction strength can be made more stable under the influence of slight changes in the beam radial thickness or position.

If the ripple period is sufficiently long, the electric field at the beam radius $R$ is given by

$$
E_{z}=A J_{o}\left(k_{c} R\right) e^{j(\omega t-k z)}+A k_{c} \Delta \frac{R}{a} J_{1}\left(k_{c} R\right) \cos \left(k_{w} z\right) e^{j(\omega t-k z)}
$$


where $J_{1}$ is the first order Bessel function. The first term can be made to vanish if the beam is centered around a null in the axial field, but it is in general nonzero.

We have used the PIC code ISIS [7] to verify the field representation in Eqns. (1) and (2). In Fig. 2 we see the coaxial geometry used in the simulation. A TM 02 mode driven at a frequency of $17.1 \mathrm{GHz}$ is used in a cylindrical waveguide with the mean diameter of the outer conductor being $3.369 \mathrm{~cm}$. The ripple period is $48 \mathrm{~cm}$ (over an order of magnitude larger than the $2.0 \mathrm{~cm}$ guide wavelength) and the ripple amplitude (peak to peak) is about $10 \%$ of the outer conductor radius. A solid inner conductor with a radius of $1.468 \mathrm{~cm}$ is additionally used, in order to suppress azimuthal modes, and to aid in experimentally driving the $\mathrm{TM}_{02}$ mode preferentially (the input matching section is not shown in this simulation). The double line in Fig. 2 at $2.92 \mathrm{~cm}$ represents the radial position we plan to inject the electron beam. In Fig. 3 we see the axial electric field at that radial position versus axial position, after Fourier decomposing the fields to remove the $e^{j(\omega t-k z)}$ factor in Eqn. (2). The field obeys Eqn. (2) very nicely over most of the rippling (the leading oscillations are probably due to mode reflections at the boundary at the axial origin due to imperfect numerical boundary conditions). By inspecting the plot, we see that the term $A J_{o}\left(k_{c} R\right)$ in Eqn. (2) is about 0.0085 (in code units) and the term $A k_{c} \Delta \frac{R}{a} J_{1}\left(k_{c} R\right)$ in Eqn. (2) is about 0.0035. Thus we have generated about a $40 \%$ ripple in the axial electric field of the mode.

In Fig. 4 we have shortened the ripple wavelength to $12 \mathrm{~cm}$. In addition, we have rippled the inner conductor with the same proportion (about 10\% peak-to-peak), but have kept all other simulation parameters constant. In Fig. 5 we show the axial electric field at $2.92 \mathrm{~cm}$, again after removing the $e^{j(\omega t-k z)}$ factor. The periodic field representation of Eqn. (2) is still fairly obvious, but with the short ripple period reflected space harmonics have been introduced. The shortest period of oscillations corresponds roughly to the ripple period divided by the number of ripples, as expected. In Fig. 6 we plot the Fourier decomposition of the axial electric field. The fundamental Fourier component (at $0.524 \mathrm{~cm}^{-1}$ ) due to the mode breathing is about eight times larger than the next largest Fourier component, which arises from the reflected space harmonics. 
In Fig. 7 we plot the axial electric field (after removing the $e^{j(\omega t-k z)}$ factor) as a function of radial position at a location where the outer conductor wall is at a minimum radius. The radial field profile obeys the radial dependency shown in Eqn. (1). The compression of the mode is very clear - the axial field smoothly drops to zero well before the mean outer radius of $3.369 \mathrm{~cm}$.

Rippling the inner conductor wall is very important for the shorter ripple periods. In Fig. 8 we plot the axial electric field (after removing the $e^{j(\omega t-k z)}$ factor) versus axial position for the case where the inner conductor is not rippled. For this case, the axial field from the compression is only twice as big as the space harmonics.

In Fig. 9 we show the longitudinal phase space for a wavelength of electrons synchronous with the mode, for a low value of $A$, and for a ten ripple periods of $6 \mathrm{~cm}$, and for a very low beam current (the purpose of this plot is to demonstrate that the interaction is identical to that of a transverse FEL). The electron trajectories in this, and the next three plots, were calculated by following the phase and energy equations of the individual electrons. The plusses correspond to the original electron phase positions and energies, and the crosses to the final electron phase positions and energy. The particles at $0,-\pi$, and $-2 \pi$ radians do not change energy, but those at other phases either gain or lose energy in the usual manner. In Fig. 10 we show the longitudinal phase space for a wavelength of electrons where the injection energy is $6 \%$ above the synchronous energy. As expected, there is a net transfer of energy from the electrons to the rf. Note that the electrons have bunched near the $-2 \pi$ phase position, and some have actually migrated to the next rf wavelength in time. In Fig. 11 we show the efficiency of power transfer from the electrons to the If (energy transferred divided by initial electron energy) as a function of electron injection energy, for six ripple periods. In Fig. 12 we have now included the effect of a large beam current driving the rf, and show the rf mode amplitude as a function of axial position. Note that the mode grows exponentially up to about $170 \mathrm{MW}$, for a beam current of $5 \mathrm{kA}$. Additionally, the maximum gain occurs for the synchronous beam energy, as seen for transverse FELs. Because the first order phase and energy differential equations for the electrons do not include the effect from the space- 
charge wave, this last plot is only approximate, but does demonstrate many features of the expected growth in the mode.

We can verify these one dimensional results by injecting particles into the PIC simulations. Explicit integration of the axial field in Eqn. (2) for the synchronous energy shows that the electrons' energy change at an axial position $z$ and at a time $t$ is given by

$$
W=A J_{o}\left(k_{c} R\right) \frac{\sin \left[\frac{z}{2}\left(\frac{\omega}{c \beta_{z}}-\beta_{1}\right)\right]_{j} j\left(\omega t-\frac{z}{2}\left(\frac{\omega}{c \beta_{z}}+\beta_{1}\right)\right)}{\frac{\omega}{c \beta_{z}}-\beta_{1}}+\frac{z}{2} A k_{c} \frac{R}{a} J_{1}\left(k_{c} R\right) e^{j\left(t-\frac{z}{c \beta_{z}}\right) \omega}
$$

where now $\beta_{z}$ is the electrons' relativistic velocity and $\beta_{1}$ is the wavenumber of the mode. Note that the first term averages to zero, and does not lead to a real energy transfer, and the second term will continually increase over distance.

In Fig. 13 we see the real space geometry used for an ISIS simulation. The if power propagates in the $\mathrm{TM}_{02}$ mode as before. There are $71 / 2$ ripples of length $12 \mathrm{~cm}$ each. The electron beam radius is at $2.92 \mathrm{~cm}$, and is confined with a $0.5 \mathrm{~T}$ axial magnetic field. There is very clear axial bunching of the beam in the axial range of $60-100 \mathrm{~cm}$.

In Fig. 14 we see the beam's axial phase space $\left(\gamma \beta_{z}\right.$ plotted versus axial position $\left.z\right)$ at a instant in time ( $0.58 \mu \mathrm{sec}$ from the start of the simulation). The prominent envelope oscillations at the start of the simulation predicted by Eqn. (2) are very clear at early axial distances, which transform into a net decrease of the beam's axial momentum at later axial distances. As in the earlier simulations, the beam current is very low, and the simulation is meant to demonstrate bunching, trapping, and energy extraction (about 10\% in this case).

\section{Phase Stability Scheme}


In this section, we discuss the major features of the phase stability mechanism. We start with the dispersion relation for the growing mode, including the influences of the beam velocity, the space-charge wave, and the detuning from resonance. After a perturbation analysis, we can determine criteria that lead to the imaginary part of the growth constant for the mode that is insentitive to beam energy.

If we assume that the growing If mode components have an $e^{j \omega t-\Gamma z}$ exponential behavior, this dispersion relation can be derived [8] for the axial FEL:

$$
\left(\left(\beta_{e}+j \Gamma\right)^{2}-\left((j \Gamma)^{2}-k^{2}\right) \hat{\beta}_{q}^{2}\right)\left(\left(\Gamma-j k_{w}\right)^{2}+\beta_{1}^{2}\right)=2 C^{3} \beta_{1}^{4}
$$

where $\beta_{e}$ is the electron propagation constant $\left(\beta_{e}=\omega / c \beta_{z}\right)$, the gain parameter $C$ is proportional to the beam current divided by $\gamma \beta^{3}$, all to the $1 / 3$ power, and the normalized spacecharge wavenumber $\hat{\beta}_{q}^{2}=4 \chi_{o} \frac{I}{I_{A}} \ln \frac{a}{R}$, where now $I$ is the beam current, $I_{A}$ is about $17 \mathrm{kA}$, and $\chi_{o}$ is a slowly varying term close to unity that depends on $a$ and $R$. Note that the real part of $\Gamma z$ is the exponential growth of the mode, and the imaginary part of $\Gamma z$ is the relative phase of the mode at the position $z$.

We can define the normalized gain $\delta$ and the detuning $\Delta$ by

$$
\begin{aligned}
& \Gamma=j \beta_{1}+j k_{w}+\delta C \beta_{1} \\
& \beta_{e}=\beta_{1}+k_{w}+\Delta
\end{aligned}
$$

Note that the phase evolution of the mode is proportional to the imaginary part of $\delta$. Eqn. (4) now becomes

$$
\left(\Delta^{2}+2 j \Delta \delta C \beta_{1}-\left(\delta C \beta_{1}\right)^{2}-\left(\left(\beta_{1}+k_{w}\right)^{2}-k^{2}-2 j \delta C \beta_{1}\left(\beta_{1}+k_{w}\right)\right) \hat{\beta}_{q}^{2}\right)(j \delta)=C^{2} \beta_{1}^{2}
$$

Now $\delta$ and thus the output phase can be made independent of beam energy if 


$$
\frac{d}{d \beta_{e}}\left(\Delta^{2}+2 j \Delta C \beta_{1}-\left(\left(\beta_{1}+k_{w}\right)^{2}-k^{2}-2 j \delta C \beta_{1}\left(\beta_{1}+k_{w}\right)\right) \hat{\beta}_{q}^{2}\right)=0
$$

for the case the derivative of the interaction strength with respect to $\beta_{e}$ vanishes. Eqn. (7) is satisfied by the conditions (for a constant perveance gun):

$$
\begin{aligned}
& \Delta=-\beta_{e} / \gamma \\
& \hat{\beta}_{q}^{2}=\frac{2}{3 \gamma(\gamma+1)\left(1-\Delta / \beta_{e}\right)} .
\end{aligned}
$$

This solution only makes sense for $\gamma$ on the order of 10 or greater because of the typically narrow window of detunings that lead to growing mode solutions. However, the first equation in Eqn. (8) is required for gain stability, and a minor modification to the second equation will lead to phase stability for small detunings.

Let us assume that $\Gamma=j \beta_{1}+j k_{w}+\delta_{o} C \beta_{1}$ is a solution of the dispersion relation, Eqn. (4). Now let us consider the solution of the dispersion relation where $\Delta$ is slightly shifted (by $\delta_{\Delta}$ ), $\hat{\beta}_{q}^{2}$ is slightly shifted (by $\delta_{\hat{\beta}_{q}^{2}}$ ), and $C$ is slightly shifted (by $\delta_{C}$ ), and where we denote the new solution by $\Gamma=j \beta_{1}+j k_{w}+\delta_{o} C \beta_{1}+\delta_{1} C \beta_{1}$. Since the solution to the growing mode has a negative real component and lags behind the electron's phase velocity, we can write $\delta_{o}=-a+j b$, where both $a$ and $b$ are positive and typically are on the order of unity. After solving for $\delta_{1}$ by performing a first-order expansion we find

$$
\hat{\beta}_{q}^{2}=\frac{2}{3 \gamma(\gamma+1)}\left(\frac{1-\frac{b \Delta}{3\left(a^{2}+b^{2}\right) C \beta_{1}}-\frac{d C / d \gamma}{C} \frac{3\left(a^{2}-b^{2}\right) C \beta_{1} \beta^{2} \gamma^{3}}{2\left(a^{2}+b^{2}\right)^{2} \beta_{e}}}{\left(1-\Delta / \beta_{e}\right)+\frac{b\left(\left(\beta_{1}+k_{w}\right)^{2}-k^{2}\right)}{2\left(a^{2}+b^{2}\right) C \beta_{1} \beta_{e}}}\right)
$$


as the condition for phase-stable operation, where the derivative of the space-charge wavenumber is with respect to the beam propagation constant.

We can numerically calculate the roots of the dispersion relation, Eqn. (4), at slightly different beam energies to find the derivative of the phase evolution of the mode with respect to beam energy. In Fig. 15 we plot the derivative of the phase evolution of the mode with respect to beam energy in the exponential growth regime versus the normalized space-charge wavenumber $\hat{\beta}_{q}^{2}$, for a case where $\gamma=2$, the frequency is $13 \mathrm{GHz}$, and with a ripple period of $6 \mathrm{~cm}$. Phasestable operation is achieved with a beam current of $5 \mathrm{kA}$ at nearly the predicted space-charge wavenumber from Eqn. (9) $\left(\hat{\beta}_{q}^{2}=0.08\right)$.

\section{Conclusion}

In this paper, we have presented both the axial FEL interaction and a scheme that will make FEL interactions of all kinds relatively phase stable. For the axial FEL interaction we have demonstrated with PIC simulations the "wiggling" of the rf mode and the interaction between the mode and the electron beam. We have additionally derived a condition for phase-stable operation starting with the axial FEL dispersion relation using a perturbation analysis. This analysis was verified by explicit calculation of the roots of the dispersion relation. Although all FEL interactions can be made phase stable with this technique, the axial FEL is probably the easiest to use, because the absence of a wiggler precludes any hidden correlations of the interaction strength with beam energy.

\section{References}

[1] M. E. Conde and G. Bekefi, "Amplification and superradiant emission from a $33.3 \mathrm{GHz}$ free electron laser with a reversed guide magnetic field," IEEE Trans. Plasma Sci., 20, p. 240, 1992. 
[2] T. J. Orzechowski, B. R. Anderson, J. C. Clark, W. M. Fawley, A. C. Paul, D. Prosnitz, E. T. Scharlemann, S. M. Yarema, D. B. Hopkins, A. M. Sessler, and J. S. Wurtele, "Highefficiency extraction of microwave radiation from a tapered-wiggler free-electron laser," Phys. Rev. Lett., 57, p. 2172, 1986.

[3] P. Volfbeyn, K. Ricci, B. Chen, and G. Bekefi, "Measurement of the temporal and spatial phase variations of a pulsed free electron laser amplifier," IEEE Trans. Plasma Sci., 22, p. 659, 1994.

[4] S. Alberti, private communication.

[5] R. A. Jong, R. D. Ryne, G. A. Westenskow, S. S. Yu, D. B. Hopkins, and A. M. Seesler, Nucl. Instrum. and Methods Phys. Res., A296, p. 776, 1990.

[6] B. E. Carlsten, "Axial free-electron laser interaction between an annular electron beam and an axisymmetric TM mode," to be published in IEEE Journ. Quant. Elect., 31, Oct. 1995.

[7] G. Gisler, M. E. Jones, and C. M. Snell, "ISIS: A new code for PIC plasma simulations," Bull. Amer. Phys. Soc., 29, p. 1208, 1984.

[8] B. E. Carlsten, "Enhanced phase stability for a Raman free-electron laser amplifier in the exponential growth regime," to be published in Physics of Plasmas, Oct. 1995. 

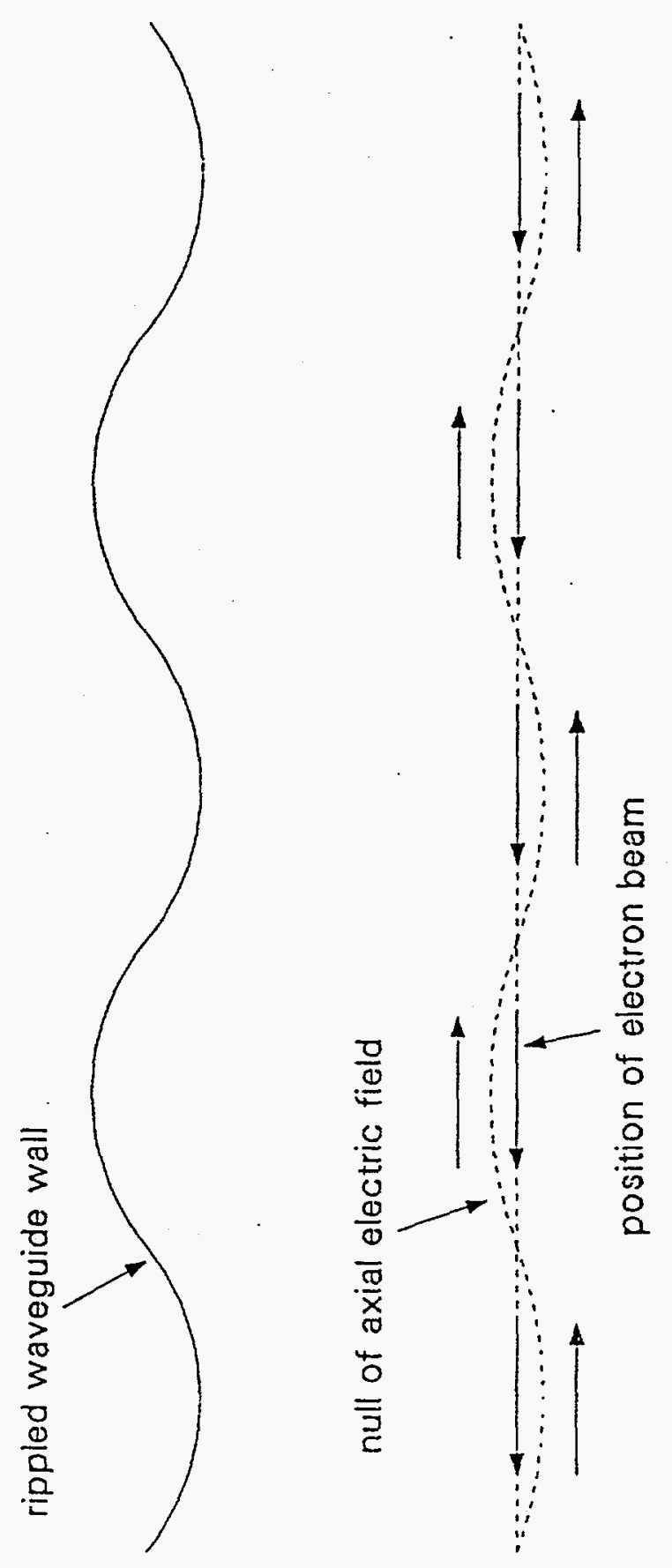


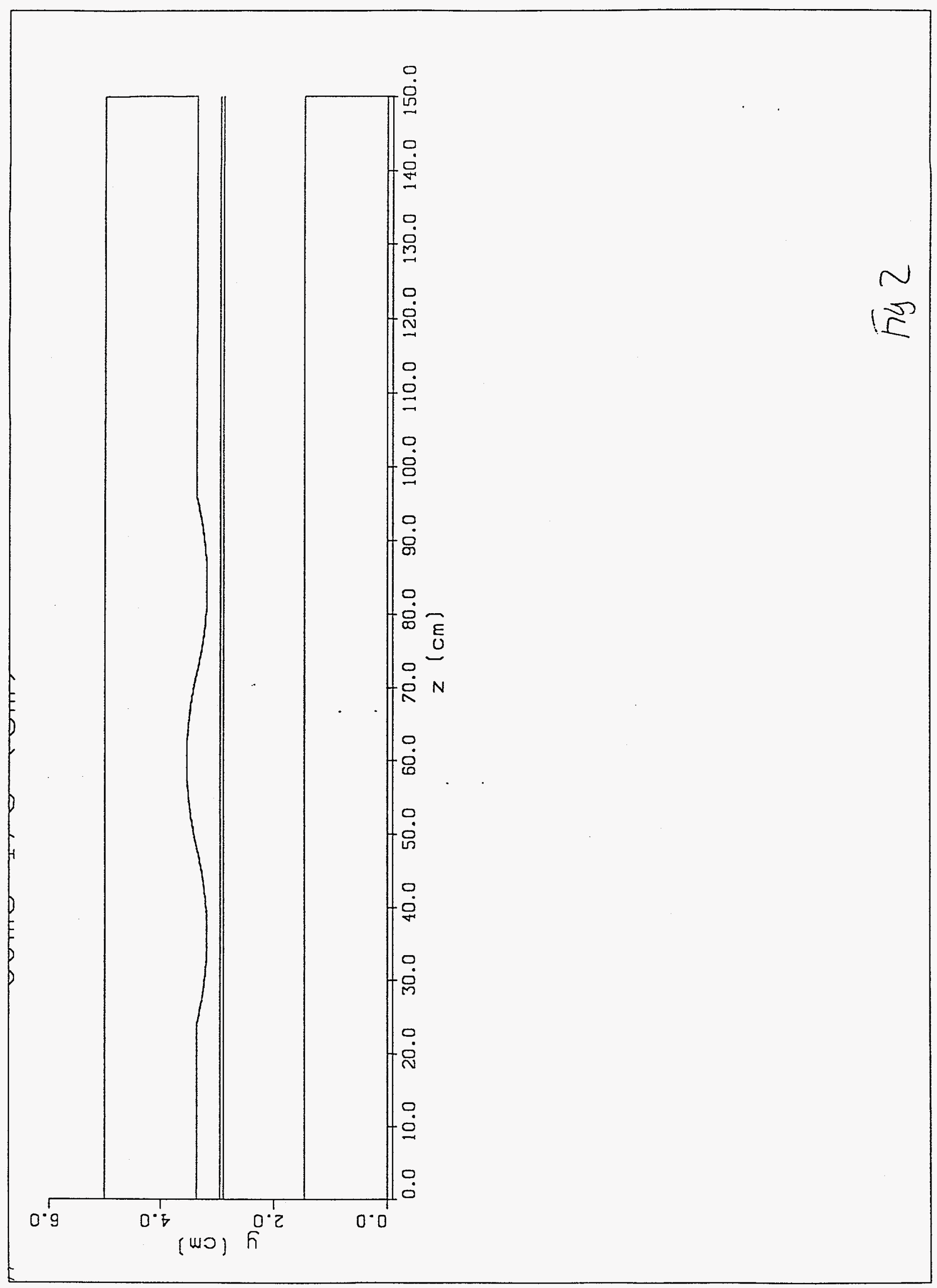




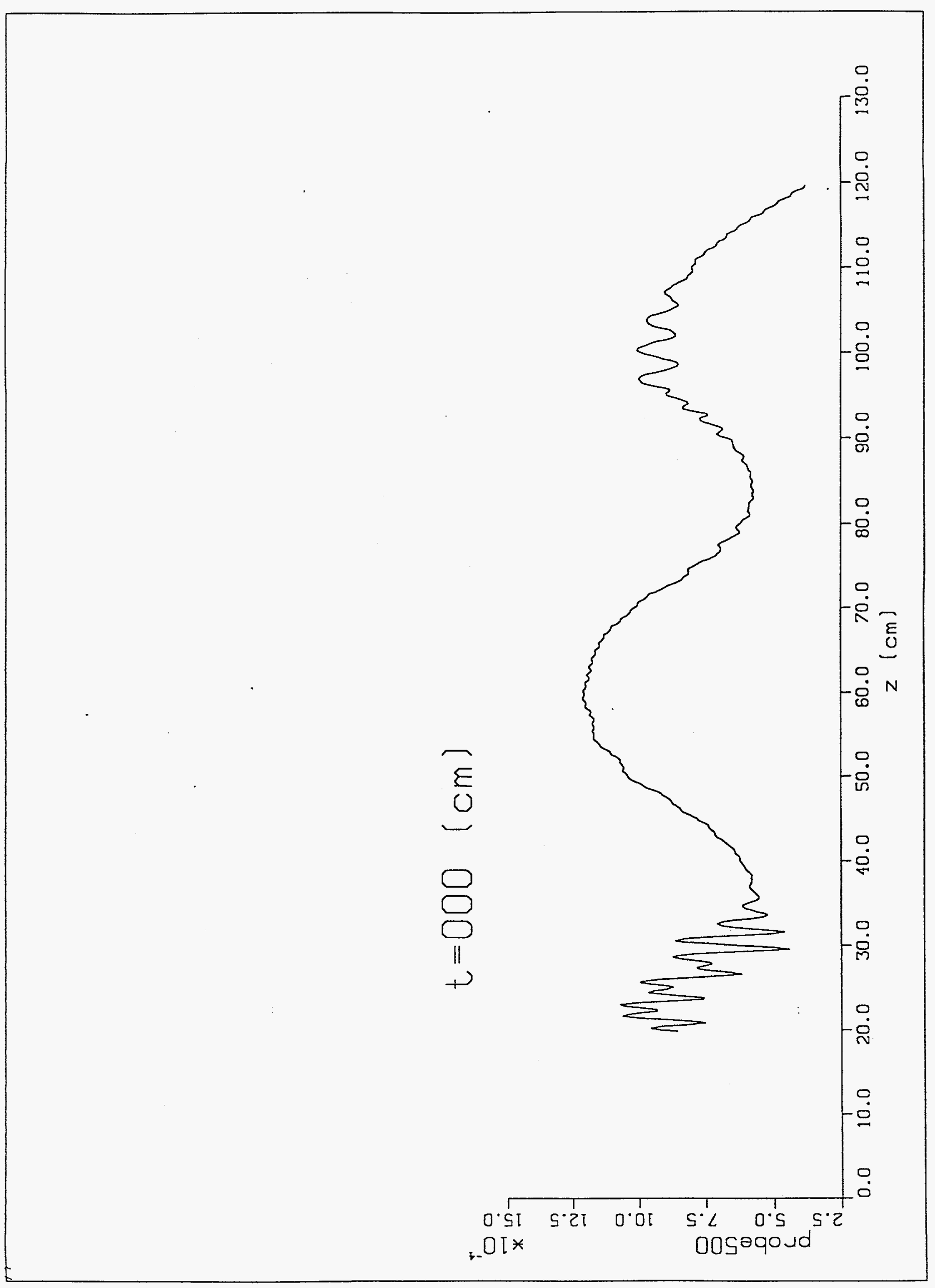




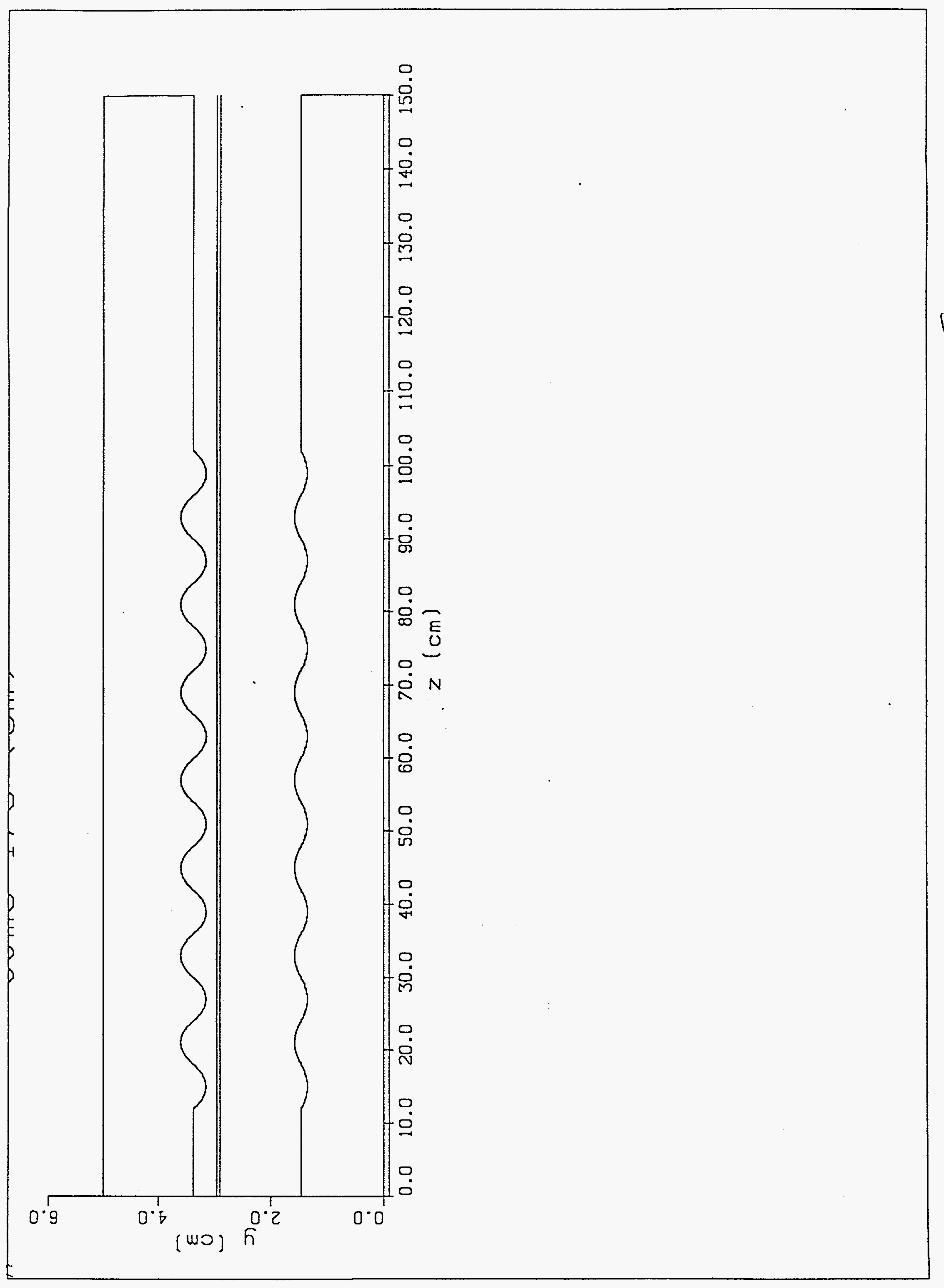




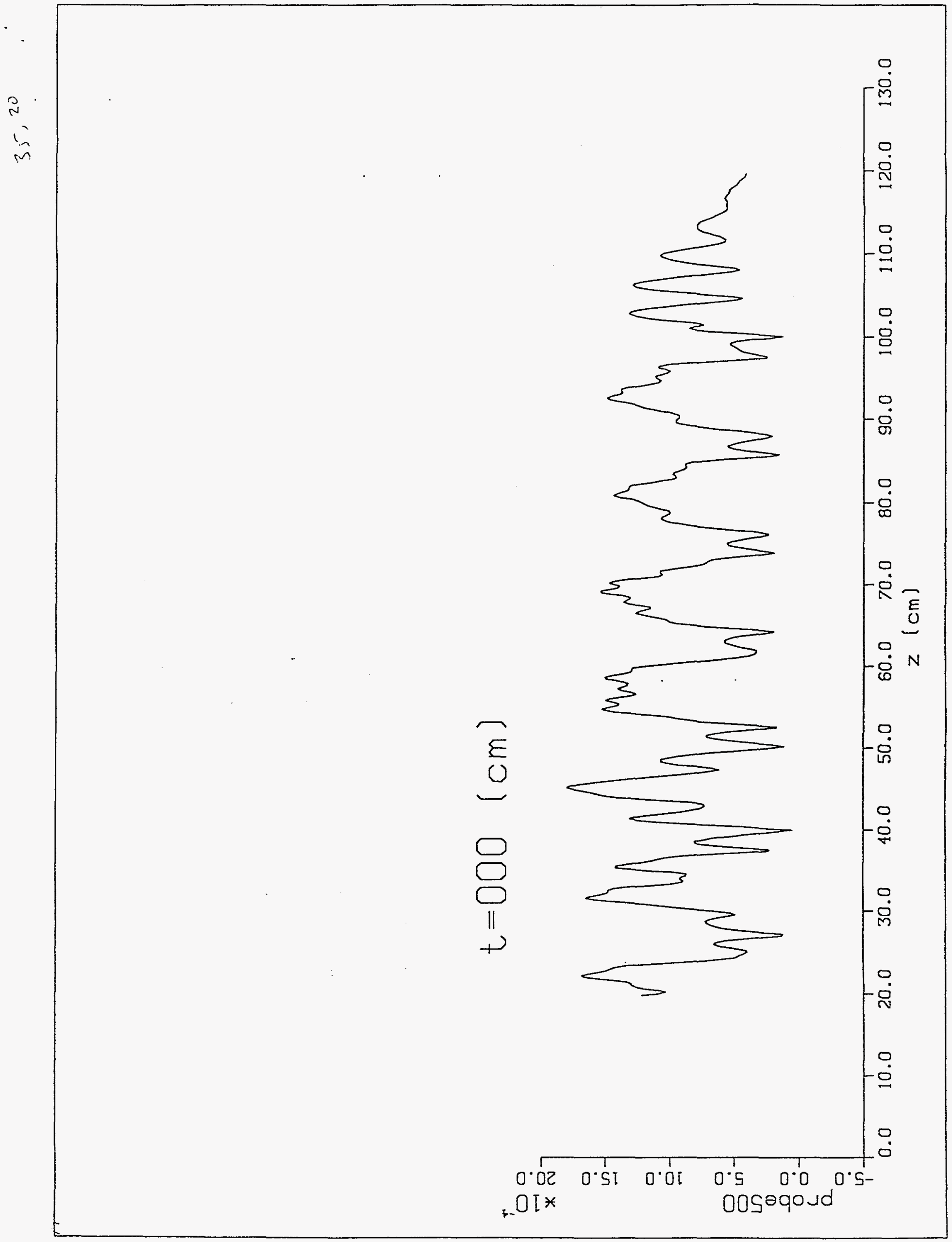




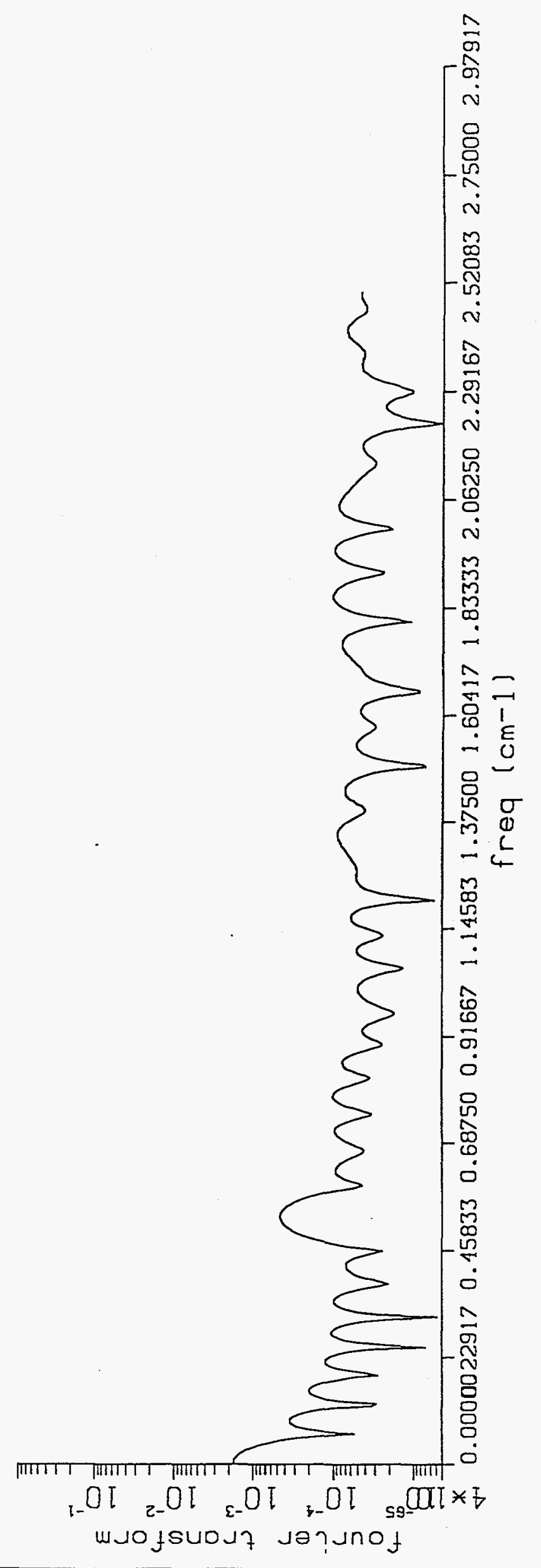




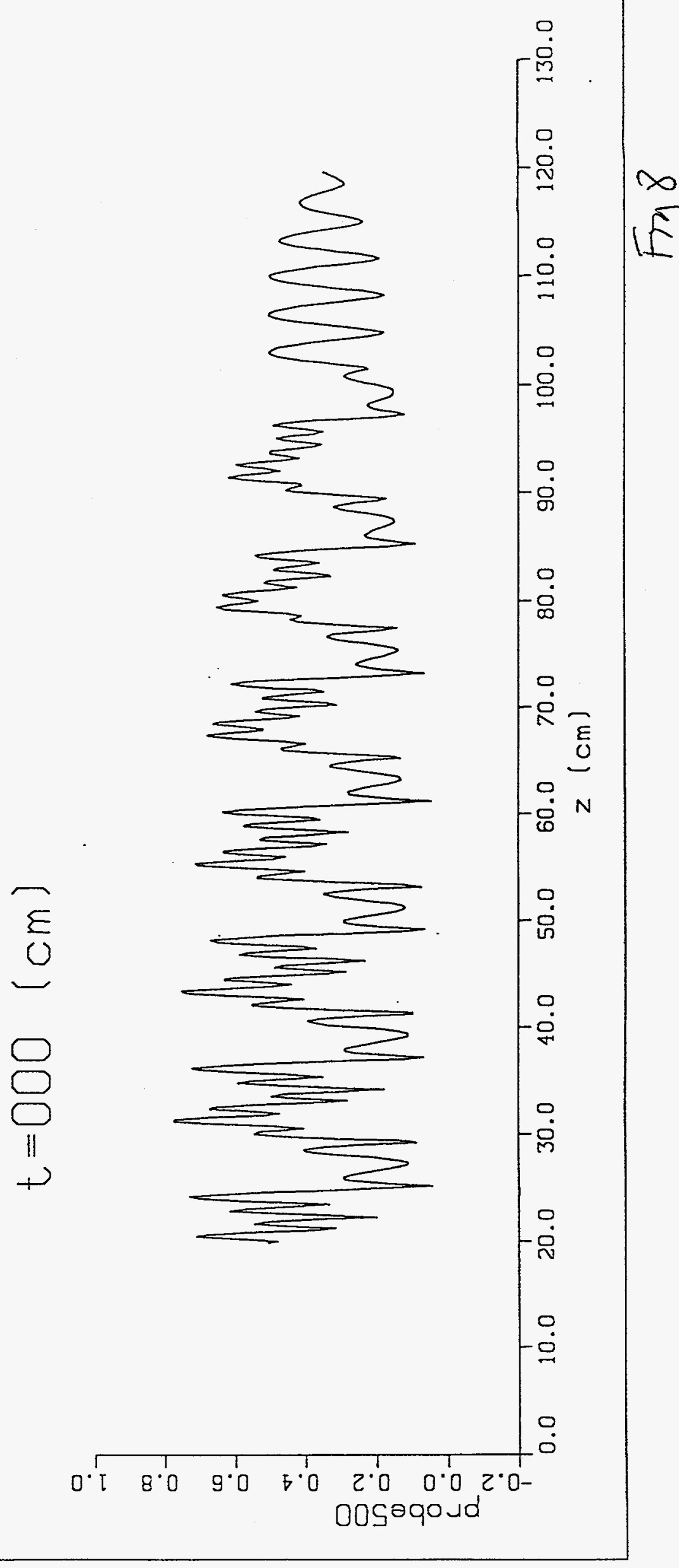



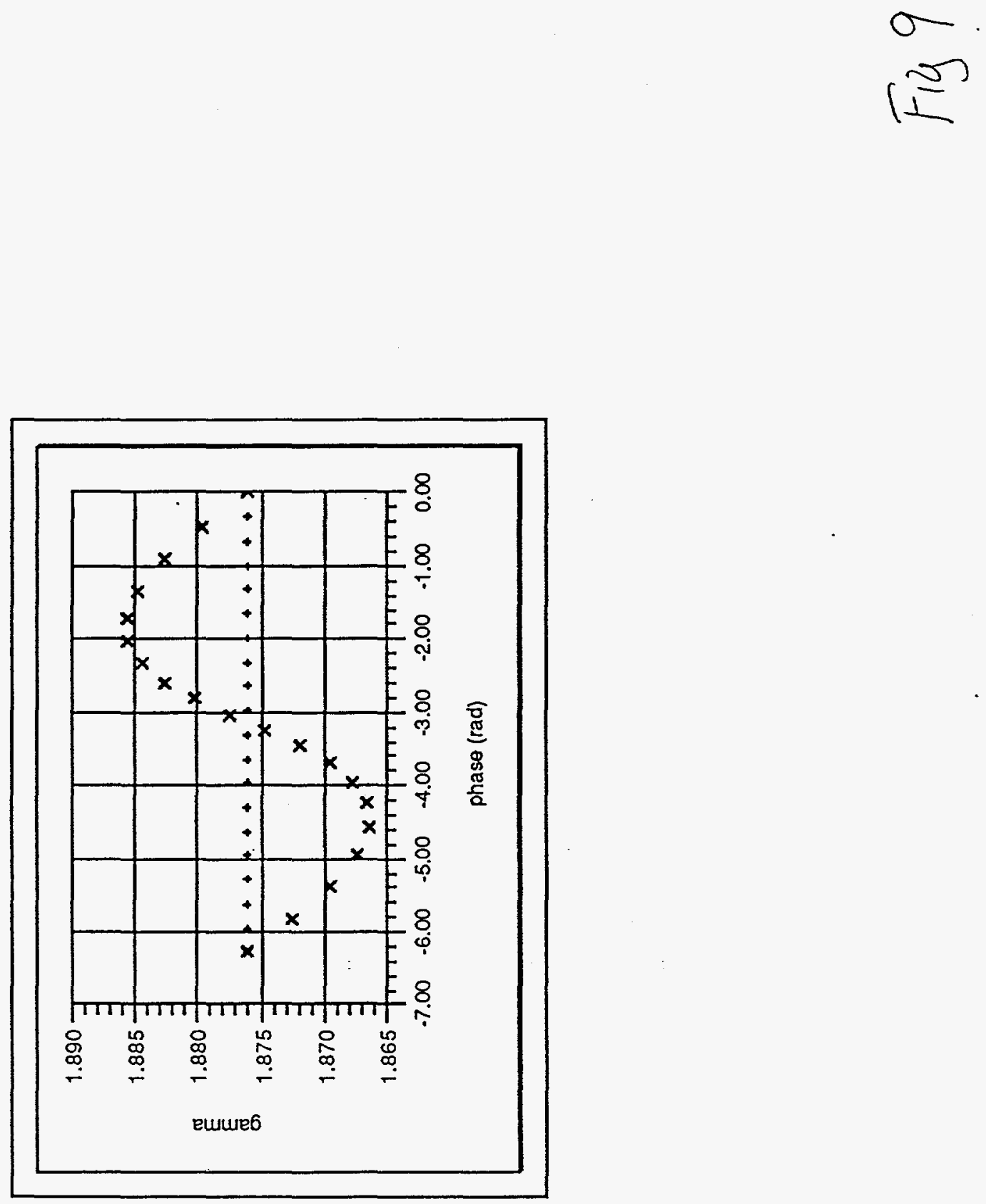


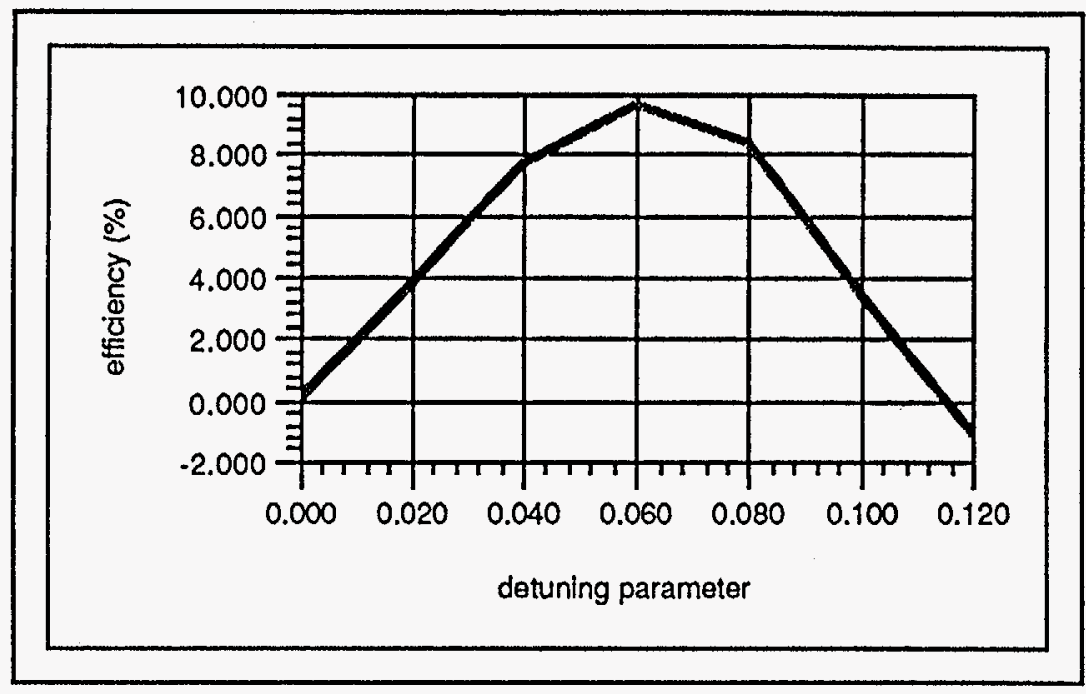

Figll

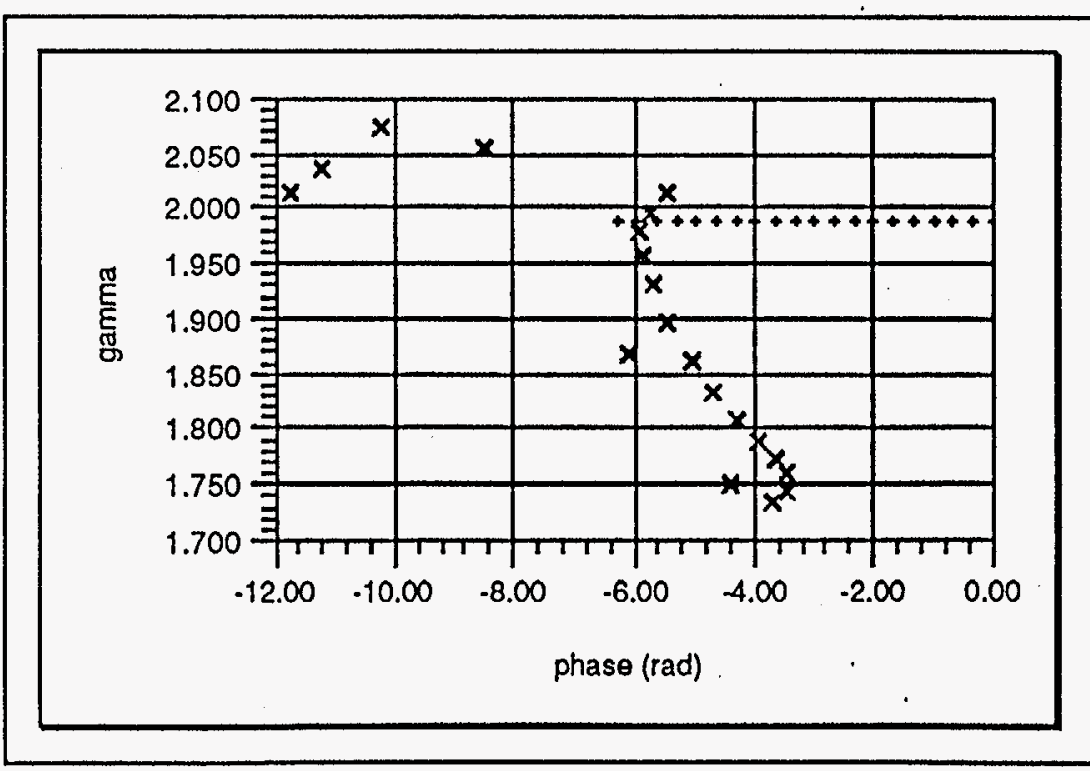



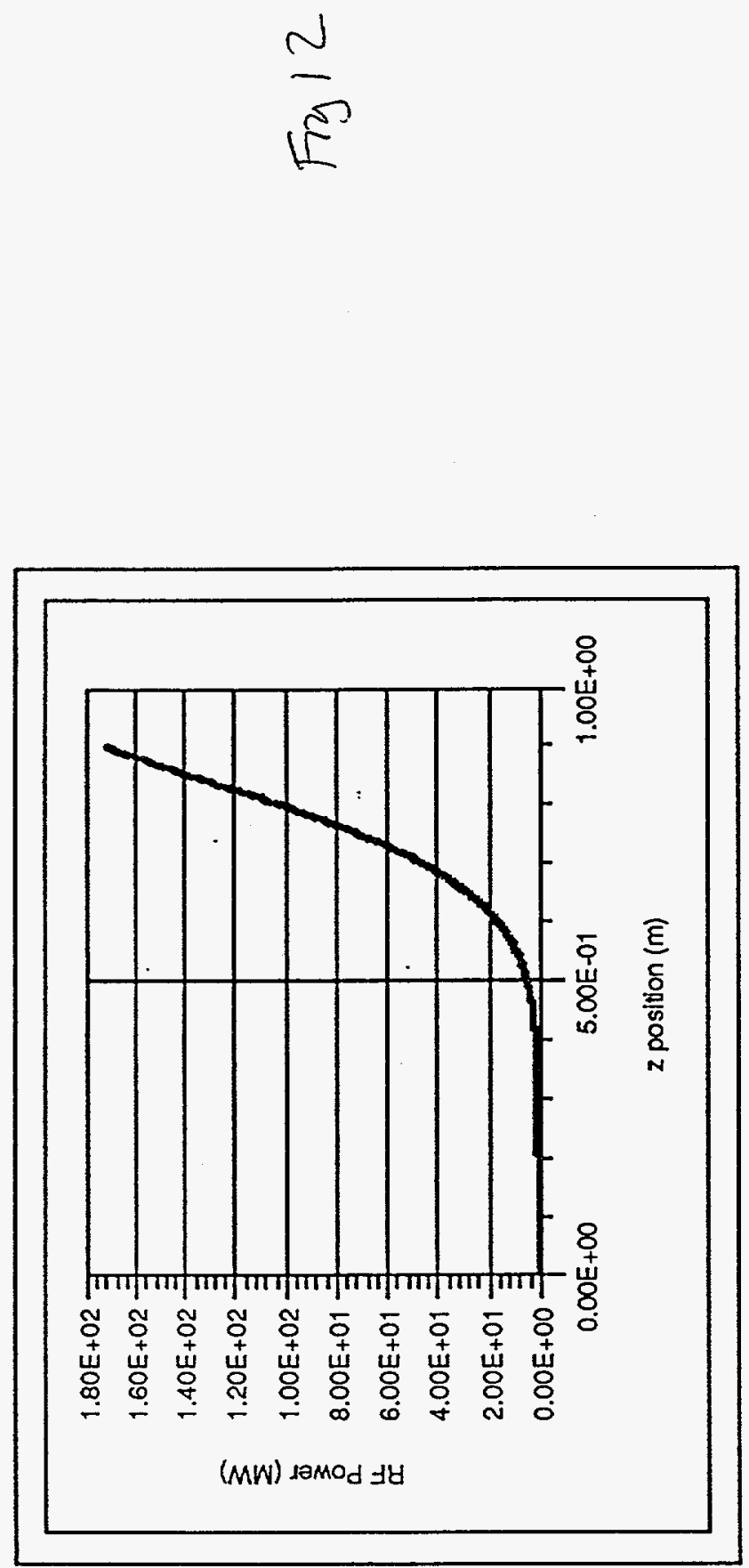


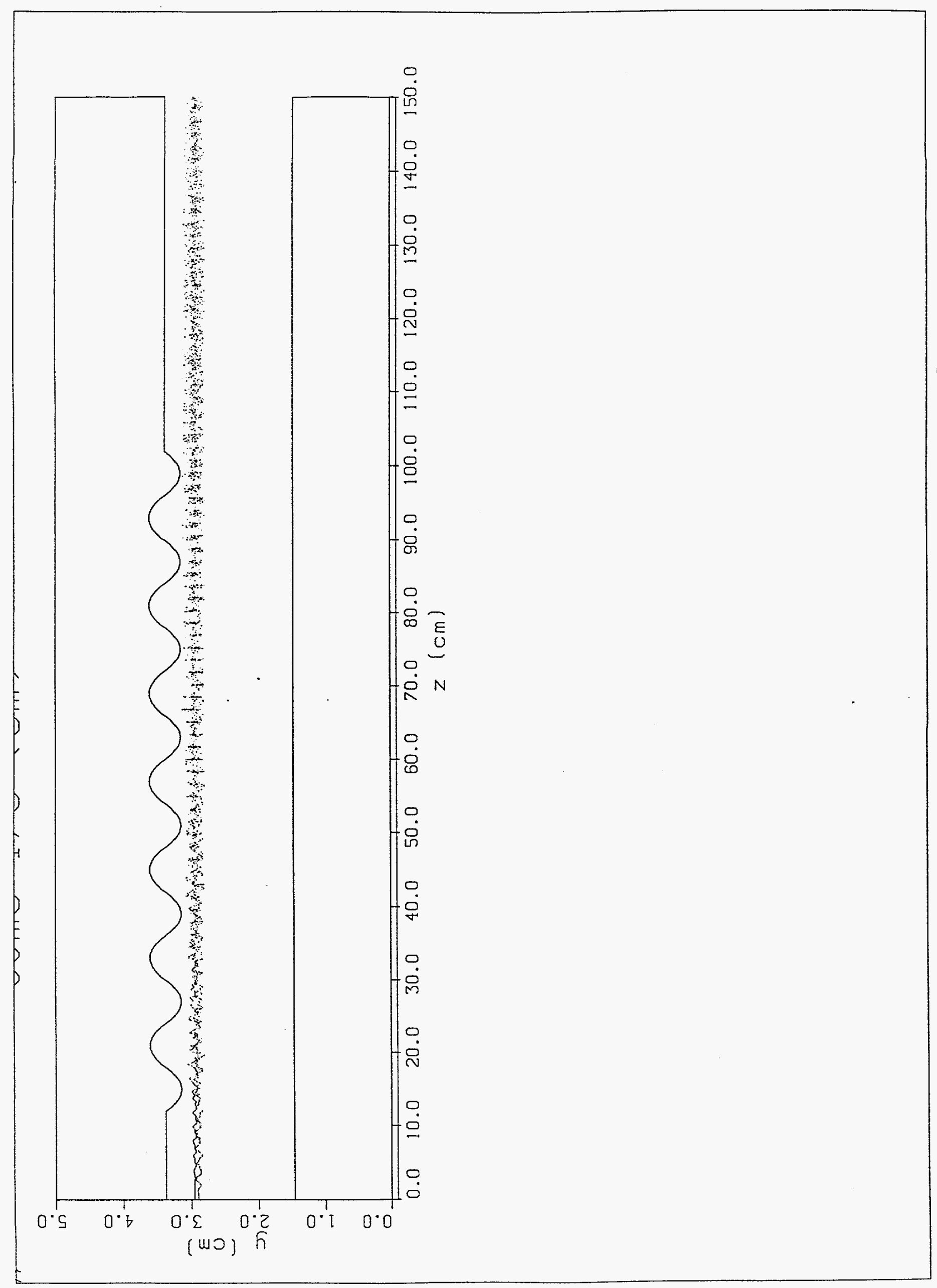




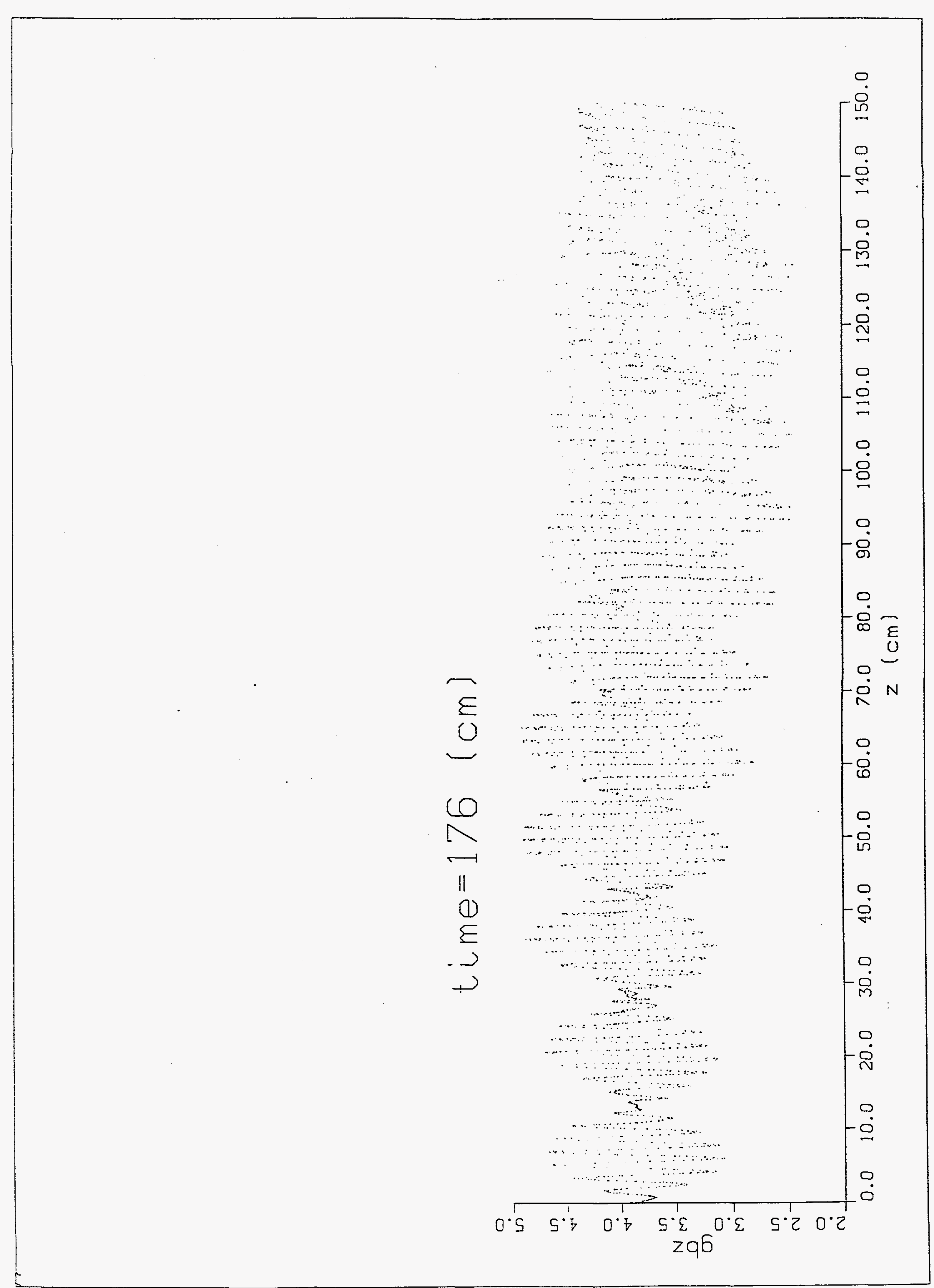




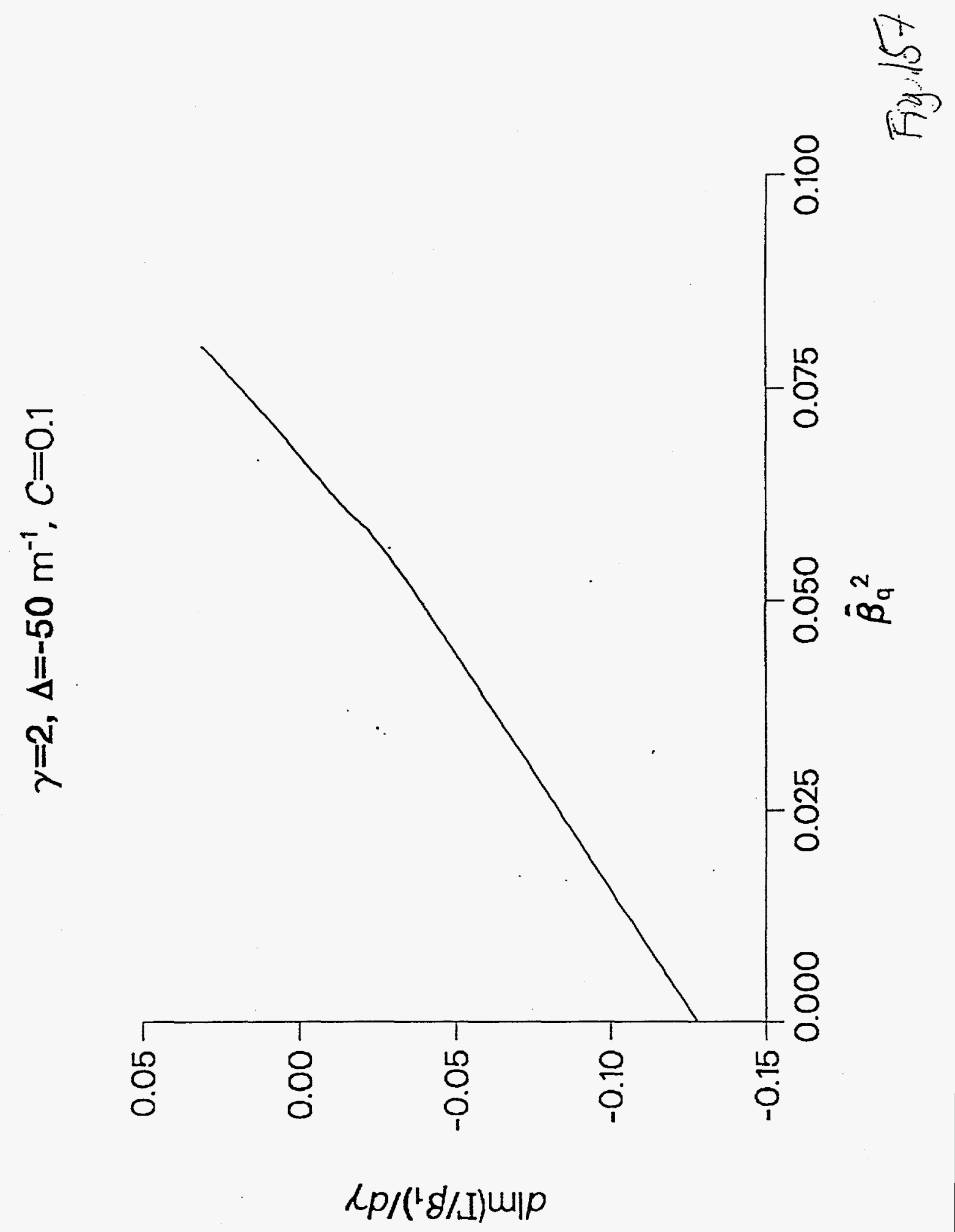

